http://jmscr.igmpublication.org/home/

ISSN (e)-2347-176x ISSN (p) 2455-0450

crossref DOI: https://dx.doi.org/10.18535/jmscr/v7i12.44

Journal Of Medical Science And Clinical Research

IGM Publication

An Official Publication of IGM Publication

\title{
Role of MRI in Evaluation of Non-Traumatic Causes of Low Backache
}

\author{
Authors \\ Dr S. Vandana Sasi Kiran MD (RD) ${ }^{1}$, Dr P. Sirisha Naidu MD (RD) ${ }^{2}$, \\ Dr Y. Trinadh M.B.B.S ${ }^{3}$, Dr T. Savitri MD (RD) ${ }^{4}$ \\ ${ }^{1}$ Assistant Professor, ${ }^{2}$ Senior Resident, ${ }^{3}$ Junior Resident \\ ${ }^{4}$ Associate Professor, Department of Radio-Diagnosis, Andhra Medical College, Visakhapatnam \\ Corresponding Author \\ Dr T. Savitri MD (RD) \\ Associate Professor, Department of Radio-Diagnosis, Andhra Medical College, Visakhapatnam
}

\begin{abstract}
Background: Low back pain is a pervading problem that affects two thirds of adults at some time in their lives. The causes of low back ache are multiple including abnormalities of the lumbosacral spine or those related to the soft tissue surrounding the lumbosacral spine. Radiological imaging plays a key role in aiding to establish or rule out pathological conditions and most importantly to influence the therapeutic decision-making process.

Materials \& Methods: The study was a prospective cross-sectional study which included 150 (based on available literature, with a confidence interval of $95 \%$ and an alpha error of 5\%) consecutive patients who underwent MR and X ray imaging in King George Hospital for non-traumatic causes of low back ache over a period of 14 months from December 2017 to January 2019.

Results: The mean age of the patients included in the study was 39.5 years. The male to female ratio was 1.38:1. The Main chief complaint is low back ache (83\%). Most common region involved is L4-L5 (81\%). Most common $x$-ray finding is spondylolisthesis (45\%). Multiple disc involvement is seen in $84 \%$ of cases on MRI.

Conclusion: The commonest age group affected by low back ache is 20-50-year. Plain radiography has a low sensitivity in detection of serious pathologies of the spine such as osteomyelitis and primary or secondary neoplasms. Magnetic resonance imaging serves as a non-invasive and highly sensitive method for evaluating the spine in the presence of low back pain.
\end{abstract}

\section{Introduction}

Low back pain is a pervading problem that affects two thirds of adults at some time in their lives. Low backache or lumbago is one of the commonest patient complaints encountered in clinical practice, having significant economic consequences. The causes of lumbago are multiple including abnormalities of the lumbosacral spine or those related to the soft tissue surrounding the lumbosacral spine ${ }^{1}$.

The disease is particularly common in daily labourers, clerks, industrial workers and software professionals and athletes. The symptomatic individuals can present with back pain or radicular pain syndrome (sciatica) ${ }^{2}$. The possible sources of pain are mechanical compression of neural elements by disc herniation, as well as direct biochemical and inflammatory causes ${ }^{3}$. Spinal 
tumors can present with a wide range of symptoms including low back ache depending on the location ${ }^{2,4}$

The main causes being muscular and ligamentous injuries, age related degenerative processes in the intervertebral disks and the facet joints. Others include spinal stenosis and disc herniation. Low back pain of musculoskeletal origin is known to occur commonly due to degenerative disorders of the lumbar spine in the absence of trauma. Disc degeneration is part of the normal ageing process and herniated discs with impingement on nerve roots can lead to radicular pain.

Plain radiography, computed tomography (CT) and MRI are the mainstay of imaging. With its high contrast and spatial resolution and lack of ionizing radiation, MRI is the best imaging technique for imaging of the spine .MR imaging provides multiplanar reconstruction and high contrast resolution for lesion characterization.

Unenhanced and contrast-enhanced MR imaging has the ability to demonstrate inflammatory, neoplastic, and most traumatic lesions as well as show anatomic detail not available on isotope studies. MR Imaging shows high sensitivity and specificity in evaluating spinal infections. With MRI evolving as the modality of choice for evaluating spinal lesions, this study aims to evaluate and categorize the non-traumatic spinal causes for low backache using MRI, an observational cross-sectional study.

Magnetic Resonance Imaging (MRI) routinely demonstrates the lumbar spine in multiple planes and extradural soft tissues (including intervertebral discs), paravertebral musculature, the exiting nerve roots and intradural structures (including the spinal cord, conus medullaris and intrathecal roots). MRI provides more complete information about all these as compared to Computed Tomography (CT) with or without intrathecal contrast agents or myelography ${ }^{5}$.

\section{Material and Methods}

The study was a prospective cross-sectional study which included 150 (based on available literature, with a confidence interval of $95 \%$ and an alpha error of $5 \%$ ) consecutive patients who underwent MR and X ray imaging in King George Hospital for non-traumatic causes of low back ache over a period of 14 months from December 2017 to January 2019.

\section{Inclusion Criteria}

All non-traumatic cases (20-69 years) of low back ache, referred to King George Hospital, Radiology department for MR imaging, during the study period.

\section{Exclusion Criteria}

1. Traumatic cases of low back ache.

2. Postsurgical Lumbosacral spine cases

3. Pregnant women

4. Age $>69$ years $\&<20$ years.

\section{Results}

The age group included in the study was 20-69 years. The mean age of the patients included in the study was 39.5 years. The male to female ratio was 1.38:1 $(\mathrm{M}=85, \quad \mathrm{~F}=65)$. Most common symptom is low back pain $(83 \%)$. Most common region involved is L4-L5 region (81\%). The commonest levels involved in multiple disc abnormalities were L4- L5+L5-S1 (35.71\%) followed by L3-L4+L4-L5+L5-S1 (17.46\%). Single discs involved in 24 cases $(16 \%)$, multiple discs involved in 126 cases (84\%). The commonest disc herniation characteristic was diffuse disc bulge $(75.33 \%)$ and disc protrusion $(39.33 \%)$.

Table 1: Age distribution of the patients

\begin{tabular}{|c|c|c|}
\hline Age group & No of patients & Percentage \\
\hline $20-29$ & 16 & $10.66 \%$ \\
\hline $30-39$ & 47 & $31.33 \%$ \\
\hline $40-49$ & & $30.66 \%$ \\
\hline $50-59$ & & \\
\hline & & $12.6 \%$ \\
\hline
\end{tabular}


Table 2: Anatomic distribution of single disc degenerative disease

\begin{tabular}{|c|c|c|}
\hline MRI levels & $\begin{array}{c}\text { Single disc } \\
\text { bbnormalities }\end{array}$ & percentage \\
\hline L1-L2 & 0 & 0 \\
\hline L2-L3 & 0 & 0 \\
\hline L3-L4 & 2 & $8.33 \%$ \\
\hline L4-L5 & 14 & $58.33 \%$ \\
\hline L5-S1 & 8 & $33.33 \%$ \\
\hline Total & 24 & \\
\hline
\end{tabular}

Table 3: Anatomical distribution of multiple discs degenerative disease

\begin{tabular}{|c|c|}
\hline MRI Levels & $\begin{array}{c}\text { Multiple disc } \\
\text { abnormalities (126) }\end{array}$ \\
\hline L3-L4+L4-L5 & 8 \\
\hline L3-L4+L5-S1 & 6 \\
\hline L4-L5+L5-S1 & 45 \\
\hline L1-L2+L3-L4+L5-S1 & 2 \\
\hline L1-L2+L4-L5+L5-S1 & 2 \\
\hline L2-L3+L3-L4+L4-L5 & 18 \\
\hline L2-L3+L3-L4+L5-S1 & 5 \\
\hline L2-L3+L4-L5+L5-S1 & 6 \\
\hline L3-L4+L4-L5+L5-S1 & 22 \\
\hline L1-L2+ L2-L3+L3-L4+L4-L5 & 1 \\
\hline L1-L2+L3-L4+L4-L5+L5-S1 & 2 \\
\hline L1-L2+ L2-L3+L3-L4+L5-S1 & 2 \\
\hline L2-L3+L3-L4+L4-L5+L5-S1 & 7 \\
\hline
\end{tabular}

Figure 1: Myxopapillary ependymoma
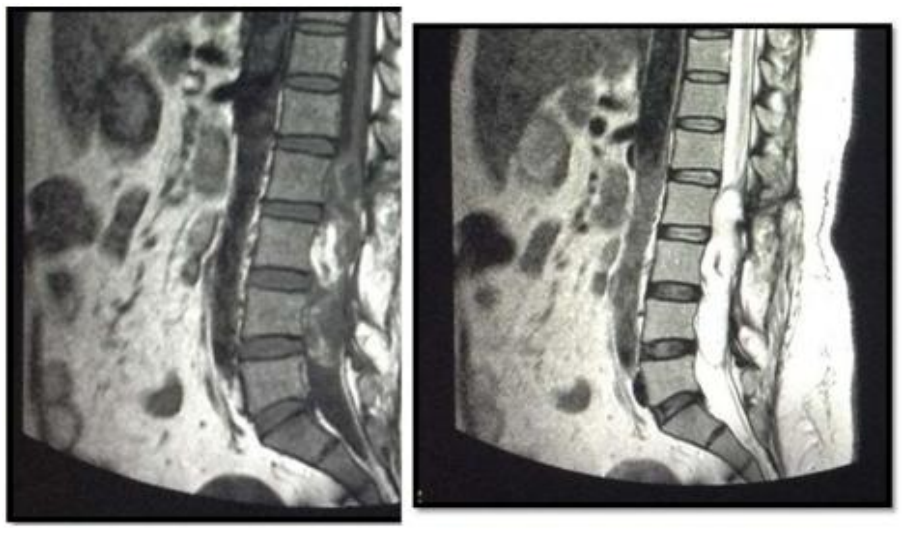

Figure 2: Perineural or Tarlov's Cyst
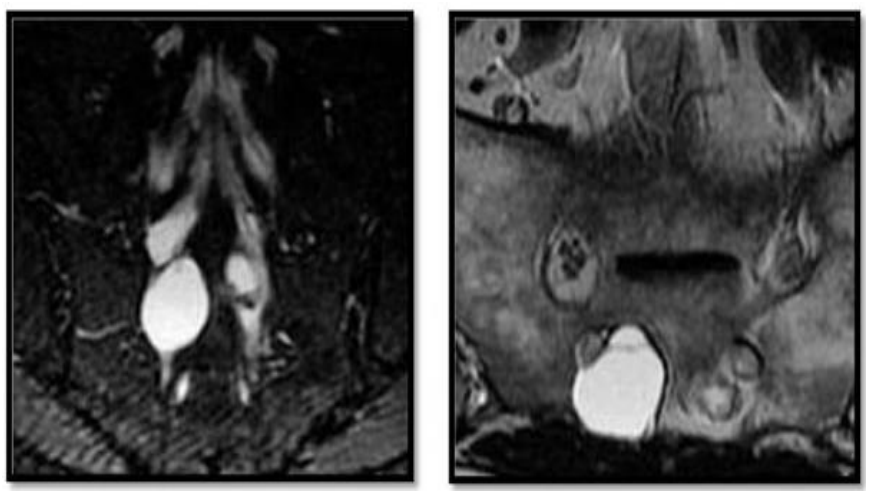

Figure 3: Anterolisthesis of L5 over S1 with poster superior migration of the disc

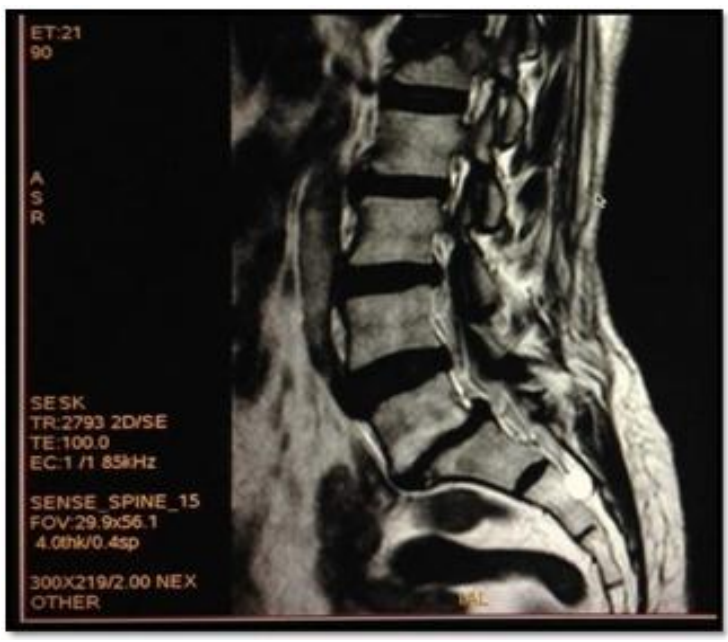

\section{Discussion}

A total of 150 patients were recruited for the study. The mean age of the study group was 2050 years. $72.6 \%$ of patients $(n=109)$ belonged to the age group of 20-50 years. This is a significant and worrisome finding as this category of patients belongs to the economically productive age group. The prevalence of disk degeneration in young individuals (20 to 39 years) could probably be explained as a result of genetic predisposition; though, other factors like repeated traumatic injuries and physical loading can play a role in causing disk degeneration. This further emphasizes the need for sensitive and refined modalities of diagnosis for early detection of disease, so as to minimize the loss of productive working hours.

\section{X- Ray Findings of Disc Degeneration}

Plain radiographs were normal in 86 of the 157case studied $(55 \%)$ on MRI examination. Disc degeneration was found in 39 of these cases $(45 \%)$. Thus, it is evident that plain radiographs have low sensitivity in detecting or predicting early disc degeneration. Similar findings were reported by pajanen et $\mathrm{al}^{6}$.

Narrowed disc space was always associated with disc degeneration in our study. Similarly end plate sclerosis was nearly all the time associated with disc degeneration. Bone erosion was seen in three cases. Two subsequently turned out to be vertebral 
osteomyelitis (Tubercular) and the third was a neoplastic lesion.

Bony canal stenosis which was seen in some cases was confirmed on MRI. Scoliosis, spondylolisthesis and transitional vertebrae were not associated with any increased incidence of disc degeneration. Findings similar to our findings have been reported by other authors.

Most of these degenerative findings were seen at L4/L5 and L5-S1 (61.2\%). Though a degenerative change of the disc begins early in life and is partly a consequence of aging, the actual cause is not known. Many factors (autoimmune, genetic and biochemical) have been implicated in accelerating the process. Since lumbar spine is subjected to heavy mechanical stress, it is a common area affected by degenerative changes. This could explain such observation in this study group. Proportion of degenerated discs progressively increases with lower spine levels, and the most common spine levels involved were L4/L5 and $\mathrm{L} 5 / \mathrm{S}^{7,8,9}$. This is similar to what was observed in this study.

\section{MRI Findings of Disc Degeneration Disc Degeneration}

At least one lumbar disc each was found degenerated in 87 cases and disc height was found reduced in 34 cases the most common level of disc degeneration was L5-S1 followed by L4- L5. Comparing with the plain radiograph findings, all cases with narrowed disc spaces on $\mathrm{X}$ - rays showed disc degeneration at the corresponding levels. Thus, a strong correlation was found between narrowed disc space and end plate changes on plain radiographs and disc degeneration at same levels on MRI.

Our findings are in consonance with the study conducted by Pajanen et $\mathrm{al}^{6}$ in 1989 , in which they compared roentgen graphic findings and disc degeneration on MRI in low back pain patients. They reported that the most common levels of disc degeneration were L5-S1 followed by L4-L5. All the cases with narrowed disc space on radiographs were associated with disc degeneration on MRI in their study. They did not specify about the correlation of end plate degeneration changes and disc degeneration on MRI in their study.

The distribution of degenerated discs in various age groups show that with increasing age the prevalence of disc degeneration increases. In 2029 -year, group $15 \%$ cases ( 3 out of 20 ) had disc generation. In 40-49 years age group 56\% cases (23 out of 41 ) had disc degeneration. All cases above 60 years of age had disc degeneration at one or more levels. Similar findings have been reported by savage et $\mathrm{al}^{10}$ and other studies reported $27 \%$ prevalence in 20-30- year age group and $52 \%$ in 31-50-year age group. Many authors believe that disc degeneration is a normal consequence of ageing and biochemical and structural changes occur simultaneously during ageing and degeneration.

\section{Disc Herniation}

Disc displacement is also a common finding in lumbar spine degenerative disease. The displaced disc can be just a simple bulge or herniation. Herniated discs can be protrusion, extrusion or sequestration. In this study disc bulging was more common (69.33\%); and this is similar to the findings reported by Sivas et $\mathrm{al}^{7,11,12}$. For herniated discs, majority ( $80 \%)$ of the herniation were protrusion, and only $20 \%$ were extrusions. Various studies have reported that disc herniation is common at L4/L5 and L5/S1 and the frequency at these levels is ranging from $30 \%$ to over $90 \%^{7,13,14,15,16}$. This was also reflected in this study as $61 \%$ of the herniated discs were at L4/L5 and L5/S1.This can be due to the large work load causing stress at these lower lumbar levels of the spine.

\section{Facet Joint Arthropathy}

In our study we found facet joint arthropathy in 31 cases $(20 \%)$. Out of these 6 revealed grades I, 17 revealed grade II and 8 revealed grade III changes. Savage et $\mathrm{al}^{10}$ reported degenerative changes in facet joints in 75 of study populations. Weishaupt 
et $\mathrm{al}^{17}$ studied asymptomatic subjects for degenerative changes in the spine and reported prevalence of mild to moderate facet joint osteoarthritis, of $18 \%$ and $22 \%$ by two separate readers. However, no severe osteoarthritis was found by either reader in their subjects. This led them to suggest that severe osteoarthritis of facet joints may play an active role in low back pain.

\section{End Plate Changes}

In our study found end plate changes in 28 cases i.e., $16 \%$ of the total study population. Largest number was found to have Type II changes (19), Type I (4) and Type III (5) changes were much less common. Modic et $\mathrm{al}^{18}$ found Type I changes in $4 \%$ and Type II changes I $16.0 \%$ cases undergoing MR imaging for lumbar disease. They did not specify the prevalence of Type III end plate changes in their study subjects. However, they further highlighted that in follow up scans of the patients with end plate changes, Type I changes were seen to convert into Type II changes represent fatty replacement of the marrow and Type III changes represent the relative absence of marrow and increase in dense woven bone as sclerosis on plain radiographs). We found evidence of associated disc degeneration at the level of involvement in all cases with end plate changes (Type I, II or III). Similar findings have been reported by Modic et $\mathrm{al}^{18}$

\section{Spinal Canal Stenosis}

In our study, we found 35 cases with spinal stenosis (a prevalence of 23.33\%). 3 cases were detected to have reduced sagittal lumbar canal diameter on plain radiographs and lumbar canal stenosis was suspected. On MRI all 3 were confirmed to have stenosis with congenitally short pedicles present and another case with bony canal stenosis was detected.

In addition, MRI revealed soft tissue canal stenosis in 4 of these cases with bony canal narrowing and in 17 other who had degenerative changes. Most of the cases had a combination of disc herniation, facet hypertrophy and ligamentum flavum hypertrophy. In two cases there was associated spondylolisthesis seen.

\section{Infective disorders}

In our study 3 cases were diagnosed to be of infective etiology. (All the cases had spondylodiscitis). On MRI a definitive diagnosis of infective spondylitis and strong likelihood of tubercular etiology was given in these cases. Contrast enhanced CT was done in all eight cases and CT guided Fine needle aspiration cytology (FNAC) was performed in one case requiring confirmation.

The cases fell in relatively younger age group, with all cases less than 50 years with a mean age of 32 years. Both the cases had presented with low back pain of insidious onset an more than two months of duration. None of the cases had fever or weight loss, though two cases reported decreased appetite. Local tenderness was elicited in three of the eight cases, straight leg raising was positive in four cases, paraspinal muscular spasm was found in five cases and lumbar mobility was restricted in seven cases. None of the case had neurological deficit but sluggish ankle jerk was found in one case.

On routine investigations ESR was marginally elevated in one case and significantly elevated in one case. Chest radiographs taken in both cases were found normal. It was observed that there was a good correlation between clinical response to treatment and MRI findings while the patients were on ATT. With appropriate therapy, the MR images revealed the reappearance of normal signal intensity (SI) of the vertebral bodies and IV discs although residual changes such as marrow replacement by fat and sclerotic changes were seen.

From our results it was suggested that MRI appearances of vertebral osteomyelitis are characteristic as reported by Modic et $\mathrm{al}^{18}$. A confluent decreased signal intensity from the vertebral bodies and intervertebral discs, with an inability to discern a margin between the disc and adjacent vertebral body on T1 weighted image, 
and increased signal intensity of the vertebral bodies and discs on T2 weighted images were reliable criteria for diagnosing vertebral osteomyelitis.

Further, multiple segment involvement, skip lesions and larges paravertebral soft tissue collections were found to be reliable predictors of tubercular etiology, as we found that the four cases treated empirically with ATT without a tissue diagnosis showed significant improvement clinically and imaging wise over three to six months confirming the diagnosis of tubercular spondylitis suggested on MRI. Sharif ${ }^{19}$ described similar findings in his reports.

\section{Spinal Neoplastic Disorders}

Intradural extramedullary lesion was found in one case, on MRI. A provisional diagnosis of nerve sheath tumors was given. The patient underwent surgery with excision of the lesions. Histopathological examination revealed neurofibroma.

According to literature nerve sheath tumors and meningiomas account for $80 \%$ to $90 \%$ of all intradural extramedullary neoplasms ${ }^{20}$. Among the nerve sheath tumors schwannomas are more common than neurofibromas, and ganglioneuromas are relatively rare. These are usually seen in middle aged adults. Multiple lesions are common with neurofibromatosis. These tumors often present with low back pain and may mimic herniation ${ }^{20}$.

An intramedullary lesion of the conus medullaris was detected in a 40-year-old female presenting with low back pain. The plain radiographs and other basic investigation ware normal. MRI revealed widened conus, with a central lesion which was isointense on T1W1 and hyperintense on T2 W1. An MRI diagnosis of myxopapillary ependymoma was made. The patient underwent surgery and excision of the lesion.

\section{Conclusion}

1) The commonest age group affected by low back ache is 20-50-year age group.
2) Degenerative disc changes are more common in L4-L5 and L5-S1 levels. Diffuse disc bulge is the commonest type of disc herniation seen on MRI.

3) X-ray showed statistically significant correlation with MRI at all levels. Degenerative disorders of the spine are the most common causes of low back pain leading to disc herniation, facet joint arthropathy, and lumbar canal stenosis.

4) Persistent low back pain may be the only presenting symptom in some serious pathologies such as vertebral osteomyelitis, metastatic vertebral lesions, primary neoplasms of vertebrae and intradural tumor with without altering biochemical/ hematological parameters or manifesting on plain radiographs.

5) Plain radiographs are not useful in early detection of most pathologies of spine including degenerative, infective and neoplastic lesions because they produce recognizable changes in plain radiographs, only in the advanced stages.

6) Magnetic resonance imaging serves as a non-invasive and highly sensitive method for evaluating the spine in the presence of low back pain.

7) Disc degeneration and other degenerative changes are the most common abnormalities found in MRI examination of cases with low back pain. Lumbar disc herniation is a common phenomenon found in such cases.

8) MRI is highly sensitive in detecting and differentiating lesions of varied etiologies such as degenerative changes infective lesions, tumors, congenital and developmental disorders, compression fractures and many other uncommon pathologies of the spine.

9) In vertebral osteomyelitis MRI shows quite characteristic appearance, facilitating a reliable diagnosis. MRI is excellent modality to detect and localize epidural collections and level of cord compressions. 


\section{References}

1. Jarvik JG, Hollingworth W, Martin B, Emerson SS, Gray DT, Overman S et al. Rapid magnetic resonance imaging vs radiographs for patients with low back pain: a randomized controlled trial. JAMA. 2003 Jun 4;289(21):2810-8.

2. Kim SH, Bak KH, Kim DW, Kang TH. Primary intramedullary spinal sarcoma: a case report and review of the current literatures. J Korean Neurosurg Soc. Nov 2010;48(5):448-51.

3. Modic T. Michael, Jeffrey S. Ross. Lumbar Degenerative Disk Disease. Radiology 2007:245,43-61.

4. Smith AB, Soderlund KA, Rushing EJ, Smirniotopolous JG. RadiologicPathologic Correlation of Pediatric and Adolescent Spinal Neoplasms: Part 1, Intramedullary Spinal Neoplasms. AJR 2012; 198:34-43.

5. Czervinke $\mathrm{H}$ : umnar IV disc disease. Neuroimag. Clin North Am 1993; 3:465486.

6. Paajanen H, Erkintalo M, Kuusela et al. Resonance study of Disc degeneration in young low back pain patients: Spine 1989; 14:982-985.

7. Ong, J Anderson, J Roche A pilot study of the prevalence of lumbar disc degeneration in elite athletes with lower back pain at the Sydney 2000 Olympic Games. Br J Sports Med 2003; 37:263-266.

8. Cheung KM, Karppinen J, Chan D Prevalence and pattern of lumbar magnetic resonance imaging changes in a population study of one thousand forty-three individuals. Spine 2009: 20;34(9):934-40.

9. Takatalo J, Karppinen J, Niinimaki J, Taimela S, Nayha S, Jarvelin MR, Kyllonen E, Tervonen O, 2009 Prevalence of degenerative imaging findings in lumbar magnetic resonance imaging among young adults. Spine 2009 July 34(16):1716- 21.
10. Savage RA, Whitehouse GH, Roberts N: The relationship between the magnetic resonance imaging appearance of the lumbar spine and low back pain. Age and occupation in males. Eur spine J 1997; 6:106-114.

11. Sivas Acar Filiz, Deniz Cılız, Uğur Erel, Esra Erkol Đnal, Kürsat Özoran, Bülent Sakman. Abnormal Lumbar Magnetic Resonance Imaging in Asymptomatic Individuals, 2009 Turk J Phys Med Rehab; 55:73-7.

12. Matijsaitis V, Vaitkus A, Pauza V, et al. Neurosyphilis manifesting as spinal tranverse myelitis. Medicina Kaunas 2006;42(5):401-405.

13. Weissleder Ralph, Wittenberg Jack, Harisinghani G. Mukesh. Primar of Diagnostic imaging, Third Edition, Copywrite C2003, Mosby, Inc, Philadelphia, Pennsylvania pg 572-4.

14. Dahnet Wolfgang. Radiology Review Manual 2007. 6th Edition, 2007 Lippincott Williams \& Wilkins. Philadelphia, Pennsylvania. Central Nervous System. Disk degenerative disease; pg 202/3/24.

15. Ducati LG, Silva MV, Brandão MM, Romero FR, Zanini MA. Intradural lumbar disc herniation: report of five cases with literature review. Eur Spine J. 2013 May;22 Suppl 3: S404-8. doi: 10.1007/s00586-012-2516-4. Epub 2012 Sep 27.

16. Shobeiri E, Khalatbari MR, Taheri MS, Tofighirad N, Moharamzad Y. Magnetic resonance imaging characteristics of patients with low back pain and those with sciatica. Singapore medical journal. 2009 Jan; 50(1):87-93.

17. Weishaupt D, Zanetti M, Hodler J, Boss $\mathrm{N}$ : MR imaging of the lumbar spine: Prevalence of intervertebral disc extrusion and sequestration. Nerve root compression, End plate abnormalities and Osteoarthritis of the facet joints in 
asymptomatic volunteers. Radiology 1998; 209:661-666.

18. Modic MT, steinberg PM, Ross JS et at: Degenerative Disc Disease: Assessment of changes in vertebral body marrow with MR imaging. Radiology 1988; 166:193199.

19. Sharif SH: Role of MR imaging on the management of spinal infections. AJR 1992; 158:1333-45.

20. Osborne AG; Tumors cysts and tumor like lesion of the spine and spinal cord. Diagnostic Neuroradiology 1994, Mosby Year book. Inc St Lousis Missouri. 